Supplemental data for:

\title{
Complement inhibitors block complement C3 opsonization and improve targeting selectivity of nanoparticles in blood
}

\author{
Hanmant Gaikwad ${ }^{1,2,3}$, Yue Li ${ }^{1,2}$, Geoffrey Gifford ${ }^{1,2}$, Ernest Groman ${ }^{1,2,3}$, Nirmal K. \\ Banda $^{4}$, Laura Saba ${ }^{5,6}$, Robert Scheinman ${ }^{2,3}$, Guankui Wang ${ }^{1,2,3 *}$, Dmitri Simberg 1,2,3 \\ ${ }^{1}$ Translational Bio-Nanosciences Laboratory, ${ }^{2}$ Department of Pharmaceutical Sciences, \\ The Skaggs School of Pharmacy and Pharmaceutical Sciences, ${ }^{3}$ Colorado Center for \\ Nanomedicine and Nanosafety, University of Colorado Anschutz Medical Campus, \\ Aurora, CO, 80045, USA \\ ${ }^{4}$ Division of Rheumatology, School of Medicine, University of Colorado Anschutz \\ Medical Campus, Aurora, CO, 80045, USA \\ ${ }^{5}$ Systems Genetics and Bioinformatics Laboratory, ${ }^{6}$ Center for Translational \\ Pharmacokinetics and Pharmacogenomics, University of Colorado Anschutz Medical \\ Campus, Aurora, CO, 80045, USA
}

${ }^{*}$ Corresponding authors 
Supplemental Table S1: Characterization of double labeled particles used in Fig. 2

\begin{tabular}{|l|l|l|l|l|}
\hline CLIO-NH2 PEG10k (60 nm) & $\begin{array}{l}\text { No. of } \\
\text { Cy3 }\end{array}$ & $\begin{array}{l}\text { No. of PEG- } \\
\text { MTz }\end{array}$ & No. of Abs used & $\begin{array}{l}\text { No. of Abs } \\
\text { conjugated }\end{array}$ \\
\hline Cy3-CLIO-PEG3400-MTz & 100 & - & - & - \\
\hline Cy3-CLIO-PEG3400-Her2-Cy5 & 100 & 2000 & 100 & 75 \\
\hline Cy3-CLIO-PEG3400-EpCAM-Cy5 & 100 & 2000 & 100 & 54 \\
\hline
\end{tabular}

\section{Supplemental Table S2: Statistical analysis for Fig. 4}

\begin{tabular}{|l|c|c|c|c|c|c|}
\hline \multicolumn{1}{|c|}{ comparison } & mean & $\begin{array}{c}\text { standard } \\
\text { error }\end{array}$ & $\begin{array}{c}\text { degrees of } \\
\text { freedom }\end{array}$ & t-statistic & $\begin{array}{c}\text { p.value } \\
\text { adjusted p- } \\
\text { value }\end{array}$ \\
\hline CLIO-PEG-EpCAM - CLIO-PEG-MTz & 66.5 & 9.64 & 190 & 6.90 & $7.45 \mathrm{E}-11$ & $3.0 \mathrm{E}-10$ \\
\hline CLIO-PEG-Her - CLIO-PEG-MTz & 120.4 & 9.64 & 190 & 12.49 & $1.59 \mathrm{E}-26$ & $6.4 \mathrm{E}-26$ \\
\hline Cy5-CLIO-PEG-EpCAM - Cy5-CLIO-PEG- & 77.0 & 9.64 & 190 & 7.99 & $1.29 \mathrm{E}-13$ & $5.2 \mathrm{E}-13$ \\
\hline Cy5-CLIO-PEGHer - Cy5-CLIO-PEG-MTz & 110.3 & 9.64 & 190 & 11.44 & $2.11 \mathrm{E}-23$ & $8.5 \mathrm{E}-23$ \\
\hline
\end{tabular}

\section{Supplemental Table S3: Statistical analysis for Fig. 6}

\begin{tabular}{|l|l|l|l|l|l|}
\hline comparison & estimate & $\begin{array}{c}\text { standard } \\
\text { error from } \\
\text { ANOVA }\end{array}$ & df & t-statistic & p.value \\
\hline Cp40 - control & -4.846 & 0.474 & 33 & -10.229 & $<.0001$ \\
\hline sCD55 - control & -2.03 & 0.474 & 33 & -4.286 & 0.0004 \\
\hline sCR1 - control & -3.559 & 0.474 & 33 & -7.512 & $<.0001$ \\
\hline & & & & & \\
\hline Cy5-CLIO-PEG-Her: & & & & & \\
\hline comparison & estimate & SE & df & t.ratio & p.value \\
\hline Cp40 - control & -4.07 & 0.474 & 33 & -8.591 & $<.0001$ \\
\hline sCD55 - control & -1.835 & 0.474 & 33 & -3.874 & 0.0014 \\
\hline sCR1 - control & -3.236 & 0.474 & 33 & -6.83 & $<.0001$ \\
\hline & & & & & \\
\hline Cy5-CLIO-PEG-MTz: & & & & & p.value \\
\hline comparison & estimate & SE & df & t.ratio & $<.0001$ \\
\hline Cp40 - control & -2.826 & 0.474 & 33 & -5.964 & 0.2365 \\
\hline sCD55 - control & -0.805 & 0.474 & 33 & -1.699 & $<.0001$ \\
\hline sCR1 - control & -2.606 & 0.474 & 33 & -5.501 & \\
\hline
\end{tabular}



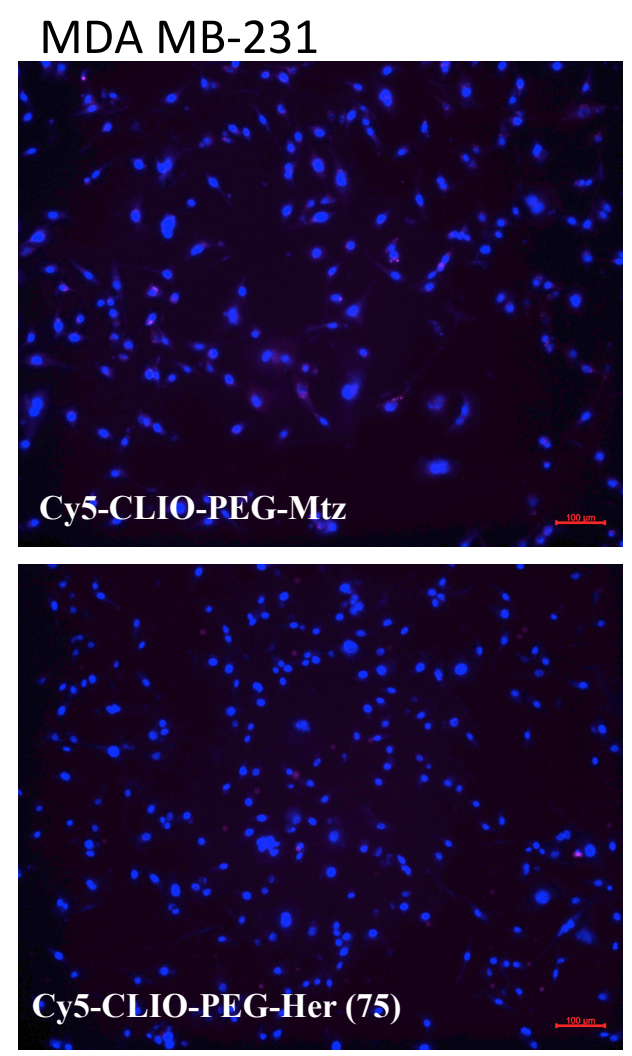

Supplemental Fig. S1: MDA MB 231 cells that do not express Her2/neu do not show any uptake of Cy5-CLIO NWs-PEG-Her. 


\begin{tabular}{|l|l|l|l|l|}
\hline CLIO NW $(60 \mathrm{~nm})$ & $\begin{array}{l}\text { No. of Cy3 } \\
\text { used }\end{array}$ & $\begin{array}{l}\text { No. of PEG-Tz } \\
\text { used }\end{array}$ & $\begin{array}{l}\text { No. of Abs } \\
\text { used }\end{array}$ & $\begin{array}{l}\text { No. of Abs } \\
\text { conjugated }\end{array}$ \\
\hline Cy3-CLIO-PEG & 500 & - & - & - \\
\hline Cy3-CLIO-PEG-Her & 500 & 10000 & 500 & 76 \\
\hline Cy3-CLIO-PEG-Her & 500 & 10000 & 300 & 32 \\
\hline Cy3-CLIO-PEG-Her & 500 & 10000 & 100 & 25 \\
\hline
\end{tabular}
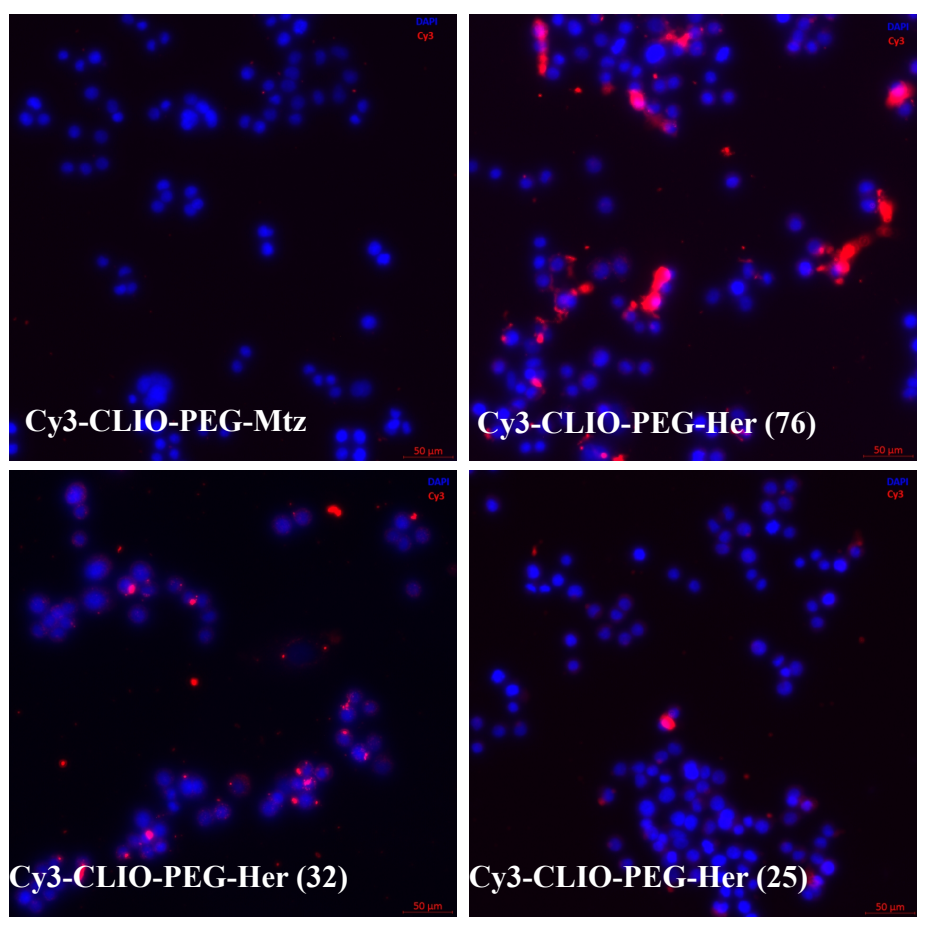

Supplemental Fig. S2: Effect of antibody/NP number on targeting efficiency. Nanoparticles were conjugated with different number of Abs (in parenthesis). CLIO NWs with higher number of antibodies achieve better accumulation in SKBR3 cells. 

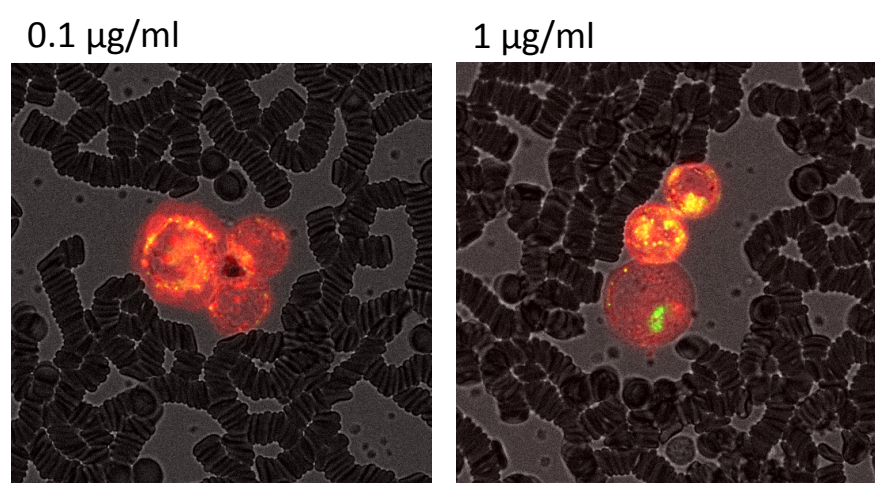

$10 \mu \mathrm{g} / \mathrm{ml}$

\section{Cy3-CLIO-PEG-EpCAM-Cy5}

Supplemental Fig. S3: Accumulation of targeted CLIO NWs in SKBR3 cells after incubation at different concentrations in whole blood. Multiple stacked erythrocytes are visible in the phase contrast. 


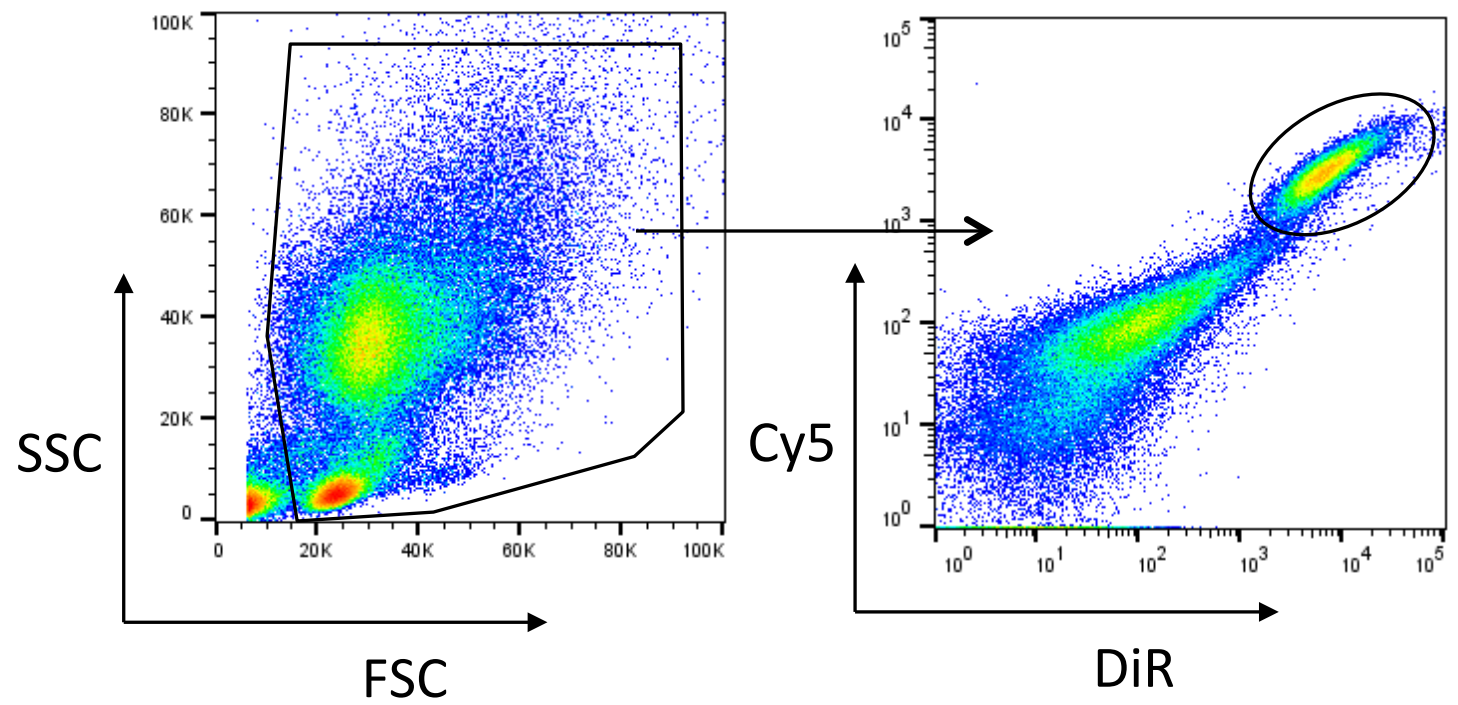

Supplemental Fig. S4: Identification of DiR labeled SKBR3 cells and Leukocytes in lysed blood.

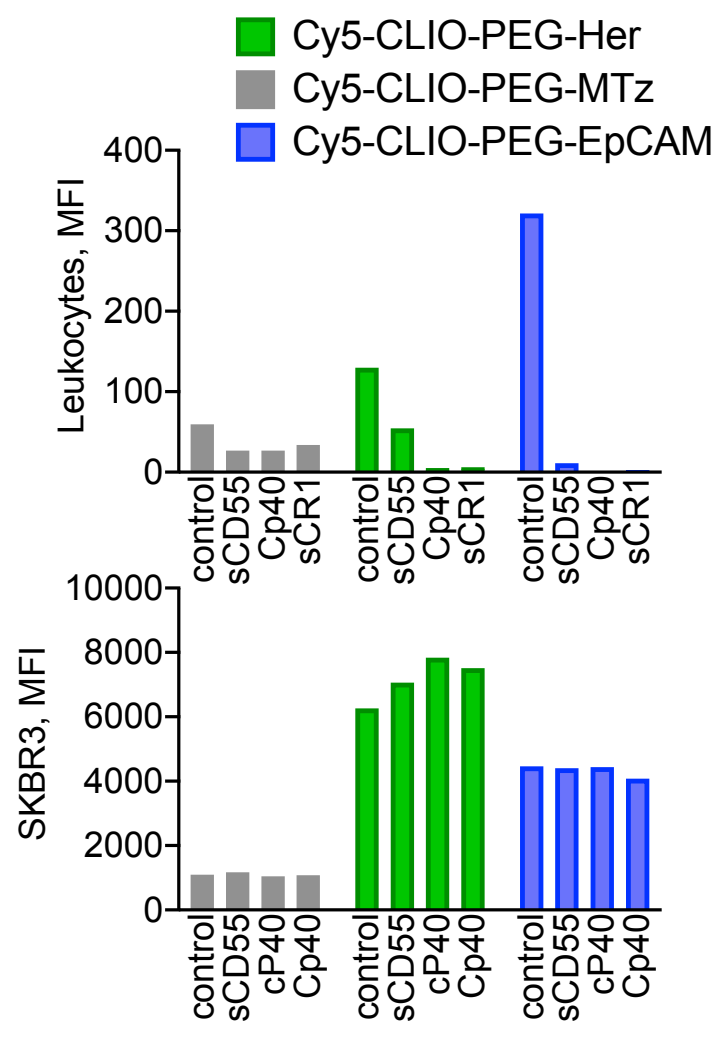

Supplemental Fig. S5: Inhibition of leukocyte uptake and no effect on SKBR3 uptake of CLIO NWs $(10 \mu \mathrm{g} / \mathrm{mL})$ by complement inhibitors. Lepirudin blood of a 67 year old male donor was used. Concentration of the inhibitors and the experiment conditions are as in Fig. 6-7 